\title{
Lack of Relationship Between Occupational Workload and Microscopic Alterations in Lumbar Intervertebral Disc Disease
}

\author{
Gerald Huschak $^{*}, 1$, Hans-Jürgen Holzhausen ${ }^{2}$, Andre Beier ${ }^{3}$, Hans Jörg Meisel ${ }^{4}$, Thomas Hoell ${ }^{3}$ \\ ${ }^{I}$ Department of Anesthesiology and Intensive Care Medicine, Medical Faculty, University of Leipzig, Germany \\ ${ }^{2}$ Department of Pathology, Martin Luther University, Medical Faculty, Halle, Germany \\ ${ }^{3}$ Spine Center Baden, Mittelbaden Hospital, Baden-Baden, Germany \\ ${ }^{4}$ Department of Neurosurgery, Bergmannstrost Hospital, Halle, Germany
}

\begin{abstract}
Objectives: The study investigated the impact of occupational workloads on disc surgery specimens. We report the relationship between workload and histological features.

Methods: Specimens were collected prospectively from patients suffering from lumbar disc prolapse $(\mathrm{n}=90)$ or spinal osteochondrosis $(\mathrm{n}=19)$. Histomorphology and occupational workload data and histomorphological features were evaluated. Occupational data were collected in a structured, standardized patient interview assessing lifting and carrying loads. In this way the exposure was assessed for each test subject's entire working life up to surgery.

Results: There was no association between cumulative workload and histological patterns. In a subgroup of patients with a workload period of 12 months prior to surgery a relevant formation of chondrocyte clusters $(\mathrm{p}=0.055)$ was apparent. Chondrocyte cluster formation was found in $83 \%(\mathrm{n}=74)$ of the prolapse patients and in $58 \%(\mathrm{n}=11)$ of the osteochondrosis patients $(\mathrm{p}=0.02)$. Fibrocyte mediated scar formation was found in $55 \%$ of the prolapse patients and in $45 \%$ of the spinal stenosis patients. Chondrocyte clusters and their de novo collagen matrix did not integrate biomechanically sufficient with collagen fibers of the disc. Disintegration of clusters from disc matrix and formation of intra-discal sequesters were observed.

Conclusion: Matrix degeneration was common but displayed no relationship to occupational workload or other histological features. Scar formation was observed in every second specimen. Regenerative chondrocyte cluster proliferation was a common feature in disc specimens and tended to be associated in patients with a workload one year before surgery.
\end{abstract}

Keywords: Chondrocyte proliferation, degeneration, histology, intra-discal scar formation, lumbar disc disease, workload.

\section{INTRODUCTION}

In Germany approximately 15,000 people per year ask for their lumbar disc prolapse to be acknowledged as an occupational disease [1]. Their request is based on the subjective experience of suffering from a heavy workload at the time the disc prolapsed occurred. A prerequisite for setting workload in the context of disc disease is biological plausibility [2,3]. Despite extensive research into the origins of lumbar disc disease, the mechanism remains unclear. The lifetime prevalence of lower back pain in the industrialized countries is $70 \%$, of which $25 \%$ appears in the form of sciatica and $1-2 \%$ as disc prolapse [4]. Degenerative changes in the disc and underlying models are well described [5-7].

The German Spine Study EPILIFT investigated the doseresponse relationship between occupational exposure to manual materials handling and/or working postures with

*Address correspondence to this author at the Klinik und Poliklinik für Anästhesiologie und Intensivtherapie, Universitätsklinikum Leipzig AöR, Liebigstr. 20, D-04103 Leipzig, Germany; Tel: +49-(0)341-97 17704; Fax: +49-(0)341-97 17709; E-mail: gerald.huschak@medizin.uni-leipzig.de trunk inclination. The EPILIFT study revealed a positive correlation between workload groups of the low and medium exposure category and disc prolapse. This finding was not seen in the highest workload category [8]. The weak correlation between heavy workload and disc prolapse supports the observation of the Ala-Kokko workgroup that occupational stress alone does not explain the occurrence of disc disease, nor do genetic factors. The combination of both factors may well contribute to disc prolapse $[9,10]$.

The biological mechanism of occupational disc prolapse is less well understood. Whereas earlier studies emphasized degeneration of the endplate as the cause of disc disease [11], more recent work does not reveal a correlation between degeneration and disc prolapse [12]. Assuming biological plausibility, a heavy workload should lead to histomorphological changes. Without this paradigm there would be no explanation for the structural changes that generate the prolapse. Recently, data from the Finnish Twin Cohort study cannot support the view that disc degeneration is a result of aging and mechanical stress or insults [13]. The phenomenon of disc degeneration is now considered to be rather genetically determined in large part [14]. The aim of our 
work was to assess the effect of lifetime workload on histomorphological intervertebral disc specimen in patients undergoing spinal disc surgery. The patients in our report were a subgroup of the EPILIFT population.

\section{METHODS}

The epidemiological study EPILIFT included 286 male and 278 female patients with lumbar disc herniation and 145 males and 206 females with lumbar chondrosis. All the patients in this study were part of the population-based multi-center case-control study which evaluated the doseresponse relationship between lifetime occupational workload and lumbar disc prolapse. Histological evaluation was performed in the subgroup of patients at the study center in Halle. Fig. (1) shows the flowchart of patient inclusion. The study was approved by the Ethics Committee of the Martin Luther University of Halle/Saale, Germany. Informed consent was obtained. For all subjects a validation of clinical and radiological diagnoses was checked by an external radiologist. Lumbar disc surgery was conducted by an open minimal invasive dorsal approach. Osteochondrosis/spinal stenosis patients were treated with decompression/revision of the spinal canal or posterior lumbar interbody fusion (PLIF). All operations were carried out by three surgeons, following a standardized surgical approach for the collection of disc material. Disc tissue was removed via a minimally invasive inter-laminar approach (without hemilaminectomy). All data in our study refer to nucleus material. The material was collected from the dorsal interior part of the disc and from the prolapse.

Workload data: The interview was based on the "German screening tool - FIT" following Karasek's job strain model $[15,16]$. It was a structured, computer-based interview. We elicited a complete occupational history, including every occupational period that lasted more than 6 months. The exposure was reconstructed for each test subject's entire working life. A cumulative dose was calculated on the basis of load weight and frequency and

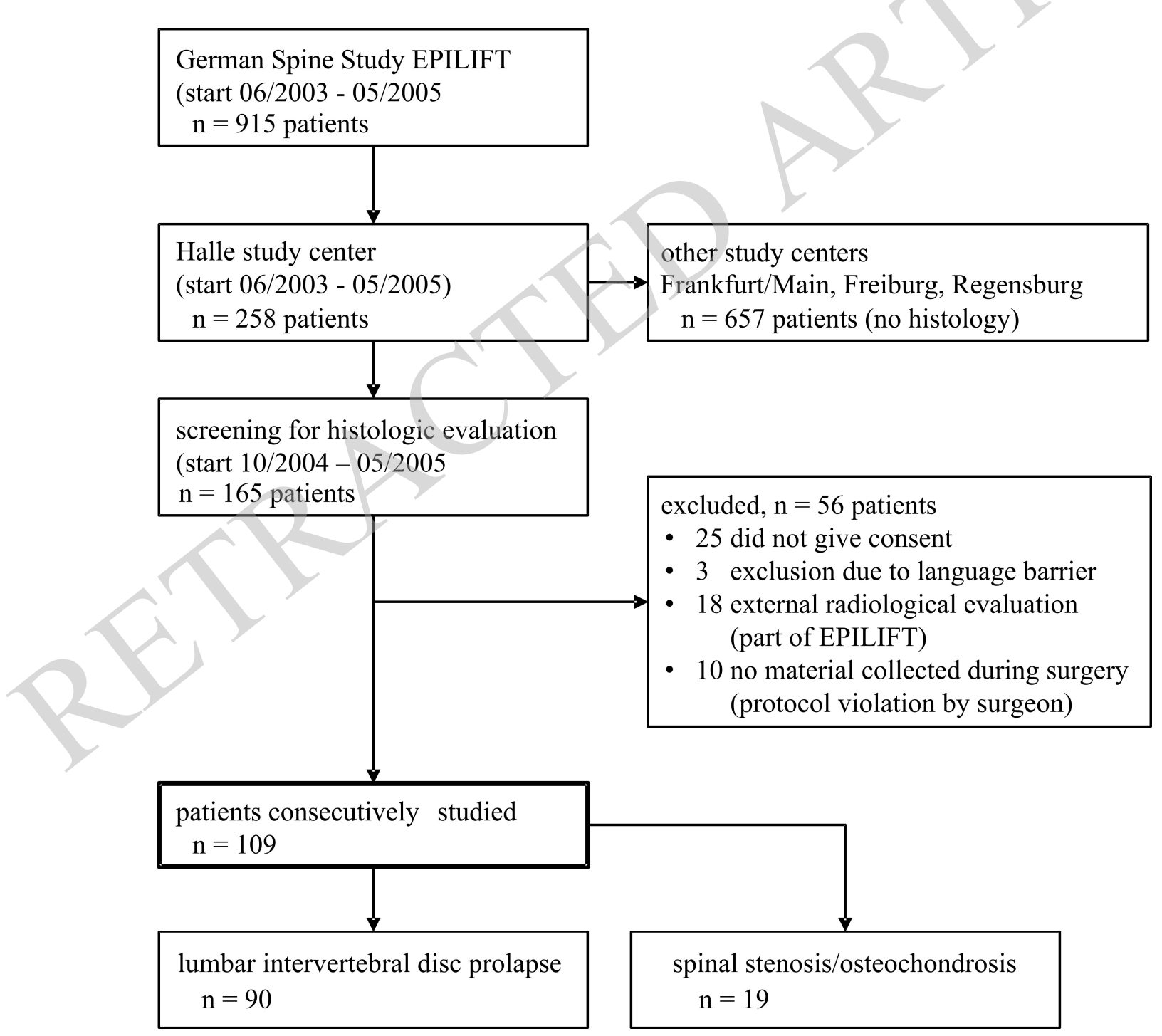

Fig. (1). The flowchart illustrates the inclusion of subjects. All the patients in this study were part of the EPILIFT study [8]. Only patients with spinal surgery were part of our investigation. 
duration of single operations with load handling. Smoking habits were recorded (never smoking, ex-smoker, or current smoker). Data on lifting and carrying of objects $>5 \mathrm{~kg}$ and duration (in h) were multiplied. Calculations were based on raw data (in $\mathrm{kg}^{*} \mathrm{~h}$ ). Out-of-work activity was not assessed.

Histology: The entire amount of disc material collected intra-operatively was used for histological evaluation. Intradiscal material from different disc areas and sequestered material were combined in a single sample. Specimen size varied between 0.5 and $2.0 \mathrm{ml}$. Disc material was fixed immediately after surgery in buffered $4 \%$ formalin solution. The paraffin block was cut in 6 steps to reduce the sampling error. Slices from each step were stained and evaluated. The data from each slice were collected at a magnification of $200 x$. The visual fields of all slices were incorporated in the evaluation. The staining was HE [Hematoxylin Eosin], PAS [Periodic acid Schiff], Alcian blue, bone morphogenetic protein antibody 2/4 [BMP 2/4] and Safranin O. The histological patterns of the specimens were evaluated microscopically (Olympus BX-41, Olympus, Germany) by two independent observer groups. Differing results were discussed in conference. Three histological features were graded per slice, using semi-quantitative scoring systems.

Regeneration: 1: single chondrocytes; 2: single chondrocyte clusters ( $>5$ clusters); 3 : numerous chondrocyte clusters. A cluster of chondrocytes was identified if more than 5 chondrocytes were agminated in a morphological formation and were surrounded by a common neomatrix shell. Chondrocyte columns appearing physiologically in the vicinity of the endplate and chondrocyte couplets in the hyaline endplate were not counted.
Degeneration: 1: absent/rarely present disintegration of the laminar fiber structure; 2: ubiquitous disintegration of collagen fibers and structural fragmentation.

Granulation, scar formation: 1: not present; 2: present.

All statistical analyses were performed on a received-totreat basis. Statistics and graphs were prepared using standard software packages (SPSS, SPSS Inc.; SigmaPlot, Systat Inc.). The stated values are means \pm standard deviation (SD). Comparisons of differences of baseline and outcome parameters were performed using the unpaired Student's $t$ test, Fisher's exact test, and the Likelihood ratiotest as appropriate. We did a graphical analysis of the factors recorded (age, body height, body weight, BMI, sex, smoking history, whole body vibration history) in relation to the three histological features of our work (chondrocyte regeneration, matrix degeneration, presence of granulation).

\section{RESULTS}

In all, 165 subjects were screened (of whom 25 patients refused to participate). Three patients were excluded because of language problems. Other 18 subjects were excluded by external radiological evaluation. In 10 cases we had no material suitable for histological examination. Inclusion flow chart is shown in Fig. (1). Baseline data are shown in Table 1. Age of the patients was $50.9 \pm 12.0$ years showing older patients in the subgroup of patients with spinal stenosis/chondrosis. A number of 61 patients $(56 \%)$ were female showing no differences between the prolapse and osteochondrosis subgroups.

Table 1. Baseline data.

\begin{tabular}{|c|c|c|c|c|}
\hline \multicolumn{2}{|c|}{ Parameter } & Prolapse $(n=90 ; 82.6 \%)$ & Osteochondrosis $(n=19 ; 17.4 \%)$ & p-Value \\
\hline \multicolumn{2}{|l|}{ Age (mean years, SD) } & $49.0 \pm 011.7$ & $60.2 \pm 8.7$ & $<0.01^{\mathrm{a}}$ \\
\hline \multirow{2}{*}{ Sex } & Female gender $(\mathrm{n}, \%)$ & $53(58.9)$ & $8(41.2)$ & \multirow{2}{*}{$0.21^{\mathrm{b}}$} \\
\hline & Male gender $(n, \%)$ & $37(41.1)$ & $11(57.9)$ & \\
\hline \multicolumn{2}{|l|}{ body weight (kg, SD) } & $84.2 \pm 14.4$ & $78.4 \pm 14.6$ & $0.11^{\mathrm{a}}$ \\
\hline \multicolumn{2}{|l|}{ height (cm, SD) } & $174.3 \pm 8.1$ & $168.3 \pm 6.5$ & $<0.01^{\mathrm{a}}$ \\
\hline \multicolumn{2}{|c|}{ Body mass index $\left(\mathrm{kg} / \mathrm{m}^{2}, \mathrm{SD}\right)$} & $27.7 \pm 4.1$ & $27.7 \pm 4.9$ & $0.99^{\mathrm{a}}$ \\
\hline \multicolumn{5}{|l|}{ Smoking habits } \\
\hline & never smoking $(\mathrm{n}, \%)$ & $45(41.3)$ & $9(8.3)$ & \multirow{3}{*}{$0.84^{\mathrm{c}}$} \\
\hline & ex-smoker $(n, \%)$ & $18(16.5)$ & $5(4.6)$ & \\
\hline & current smoker $(\mathrm{n}, \%)$ & $27(24.8)$ & $5(4.6)$ & \\
\hline \multirow{5}{*}{ Spinal level ${ }^{\mathrm{d}}(\mathrm{n}, \%)$} & $\mathrm{L} 1 / \mathrm{L} 2$ & 0 & $1(5.3)$ & \multirow{5}{*}{$<0.01^{\mathrm{c}}$} \\
\hline & $\mathrm{L} 2 / \mathrm{L} 3$ & $3(3.3)$ & 0 & \\
\hline & $\mathrm{L} 4 / \mathrm{L} 4$ & $4(4.4)$ & $5(26.3)$ & \\
\hline & L4/L5 & $45(50.0)$ & $12(63.2)$ & \\
\hline & $\mathrm{L} 5 / \mathrm{S} 1$ & $38(42.4)$ & $1(5.3)$ & \\
\hline
\end{tabular}


Table 2. Histological data for patients with lumbar intervertebral disc prolapse or lumbar osteochondrosis.

\begin{tabular}{|c|c|c|c|c|c|}
\hline & Histology $(\mathrm{n}, \%)$ & Prolapse $(n=90)$ & Osteochondrosis $(n=19)$ & \multicolumn{2}{|c|}{ p-Value } \\
\hline \multirow{3}{*}{ 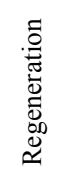 } & single chondrocytes & $15(16.9)$ & $8(42.1)$ & 2 & \multirow{3}{*}{0.028} \\
\hline & single chondrocyte clusters ( $>5$ clusters) & $48(53.9)$ & $5(26.3)$ & & \\
\hline & numerous chondrocyte clusters & $26(29.2)$ & $6(31.6)$ & & \\
\hline \multirow{2}{*}{ 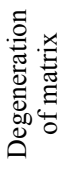 } & absent/rarely present & $35(39.8)$ & $4(21.1)$ & \multirow[t]{2}{*}{$د$} & \multirow{2}{*}{0.112} \\
\hline & ubiquitous disintegration & $53(60.2)$ & $15(78.9)$ & & \\
\hline \multirow{2}{*}{ 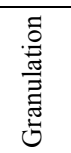 } & present & $47(55.3)$ & $8(44.8)$ & & \multirow{2}{*}{0.402} \\
\hline & not present & $38(44.7)$ & $10(55.6)$ & & \\
\hline
\end{tabular}

The table shows the histological data for disc prolapse and osteochondrosis patients. Prolapse patients showed more chondrocyte formations than chondrosis patients. Both groups had similar grading of degeneration and scar formation/granulation. P-value (Chi-Square Likelihood quotient) is stated for differences between prolapse and chondrosis patients.

Grading of histological patterns is shown in Table 2. Single chondrocyte clusters (grading 2) or numerous clusters (grading 3) were found in $83 \%$ of the prolapse specimens and in $58 \%$ of the osteochondrosis patients. Chondrocyte clusters were more frequent in disc prolapse than in osteochondrosis $(\mathrm{p}=0.034)$. A typical example of chondrocyte cluster formations in a prolapse patient is shown in Figs. $(\mathbf{2}, \mathbf{3})$. They grow in the vicinity of discal clefts. The median cluster size of all groups was $6.53 \mu \mathrm{m}$. Clusters grew to a certain size but never displayed uncontrolled growth. The smallest cluster appeared in a specimen with little chondrocyte regeneration. The largest cluster size was found in a specimen with a large amount of chondrocyte proliferation. There was no association between the level of spinal surgery and the histology pattern.

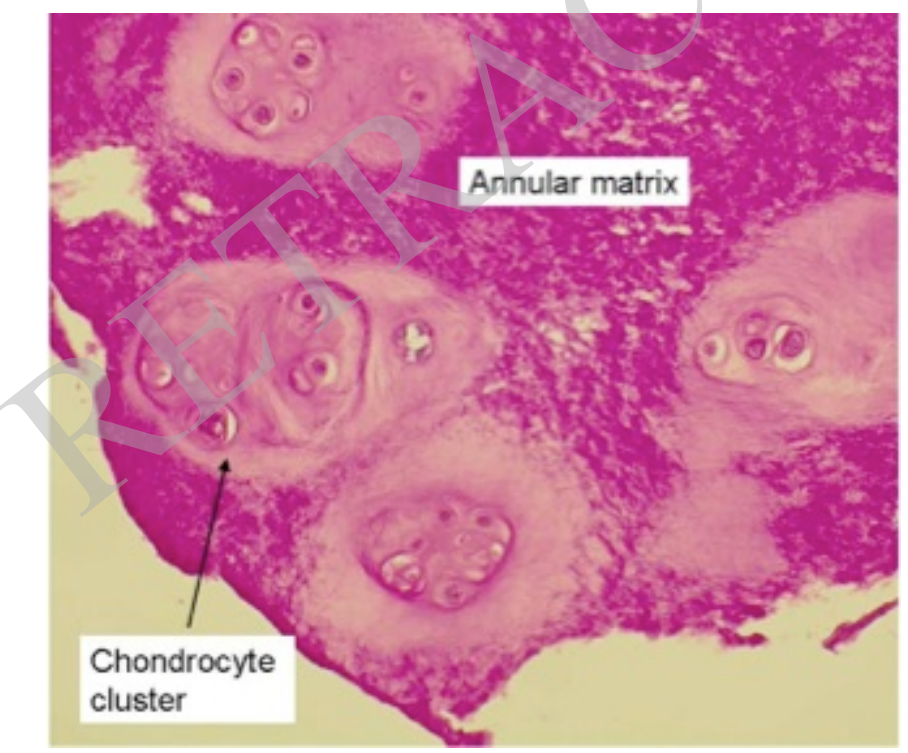

Fig. (2). The figure shows chondrocyte clusters embedded in preexisting annular matrix. The clusters display neo-matrix production. The neo-matrix and the preformed matrix are demarcated. PAS, 200x.

The expression of growth factors in specimens with frequent cluster formation revealed a distinct growth pattern. In juvenile clusters with few cells and a small rim of neo- matrix, bone morphogenetic protein heterodimetic (BMP) $2 / 4$ is expressed in virtually all cells. Large adult clusters displayed a mixture of vital and non-vital cells. Only the vital cells expressed BMP 2/4. Neo-matrix of cluster and preformed matrix were distinguishable in all specimens because of the homogenous fiber alignment of the neomatrix that did not show signs of degeneration. Cluster with more cells showed in general a larger surrounding neo matrix area. Adult clusters with a high percentage of non-vital cells were disintegrated from the ambient matrix

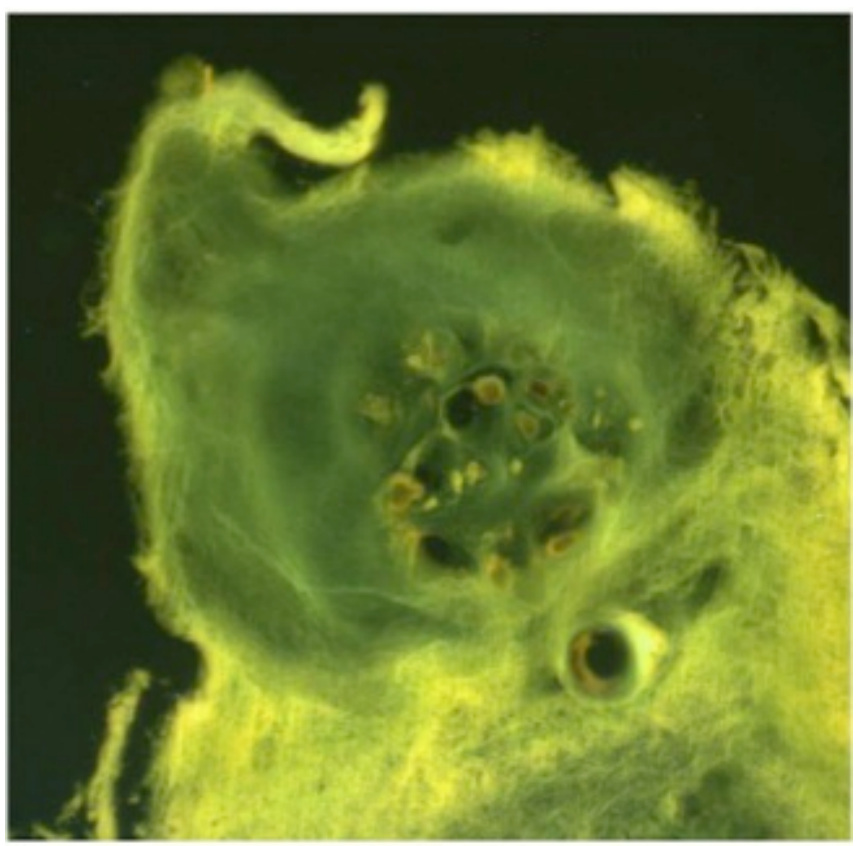

Fig. (3). Single chondrocyte cluster from a disc prolapse specimen. The lower part of the cluster is connected to the pre-existing matrix. The upper part is disintegrated from the matrix due to degenerative changes. HE, UV $407 \mu \mathrm{m}, 400 x$.

and showed a tendency to form intra-discal sequesters (Fig. 4). Younger patients displayed more clusters than older ones $(\mathrm{p}=$ 0.034 , subgroup separation at the median age of 49.5 years). 
The cells inside a cluster were strongly connected but did not build syncytial agglomerations. Smoking did not correlate with any of the histological parameters investigated. All specimens displayed matrix degeneration with a tendency towards more degeneration in the osteochondrosis patients $(\mathrm{p}=0.11)$. Granulation as a sign of intra-discal scar formation was found in $55 \%$ of the prolapse patients (Fig. 5) and in $45 \%$ of the osteochondrosis patients $(\mathrm{p}=0.40)$ (Table 2). Scar formation did not correlate with degeneration.

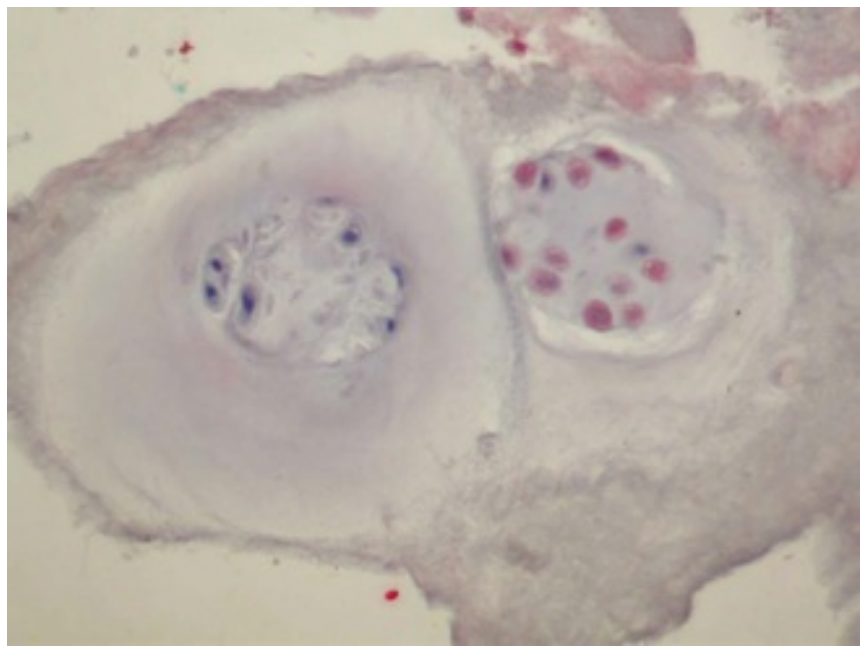

Fig. (4). The image shows two clusters, a small one with extensive BMP $2 / 4$ expression at the right side and a larger one with poor BMP 2/4 expression on the left side. Because of high BMP expression, vital cells and a small rim of neo-matrix the right cluster was considered to be a young cluster. The left one with the large amount of non-vital cells, poor BMP expression and the large rim of neo-matrix was considered to be a mature cluster (BMP 2/4, 400x).
The mean total lifetime workload of lifting and carrying forces was $\log 2.21 \pm 1.63 \mathrm{~kg} * \mathrm{~h}$ for all patients. The prolapse group showed $\log 2.24 \pm 1.60 \mathrm{~kg} * \mathrm{~h}$; the osteochondrosis group had $\log 2.09 \pm 1.81 \mathrm{~kg}^{*} \mathrm{~h}$. There was neither an association between the workload and degeneration nor between workload and scar formation. The results showed that heavy workloads tended to increase the formation of regenerative clusters in prolapse patients (single chondrocytes: $\log 2.02 \pm 1.66 \mathrm{~kg} * \mathrm{~h} ; 5$ or more chondrocyte clusters: $2.17 \pm 1.7 \mathrm{~kg} * \mathrm{~h}$; numerous chondrocyte clusters $2.47 \pm 1.44 \mathrm{~kg} * \mathrm{~h} ; \mathrm{p}=0.64)$. Frequency plots are shown in Fig. (6). None of the factors recorded (age, body height, body weight, BMI, sex, smoking history, or whole body vibration history) tended to influence the three histological features of our work (chondrocyte regeneration, matrix degeneration, presence of granulation).

The subgroup of patients suffering from workload exposure in the last year before surgery was selected for separate analysis of workload impact. This group revealed a nearly significant relationship between chondrocyte cluster formation and workload (Table $3 ; p=0.055$ ). This finding was the only histological parameter associated with workload.

\section{DISCUSSION}

The aim of the study was to elucidate the relationship between histological patterns and occupational workload in lumbar disc surgery patients. Histopathology focused on chondrocyte cluster formation. Chondrocyte clusters were known to evolve in the vicinity of intra-discal clefts. One question was whether they reflected the tissue response to mechanical disc trauma more specifically than other changes like matrix degeneration and intra-discal scar formation. The comparison of the cumulative occupational workload with

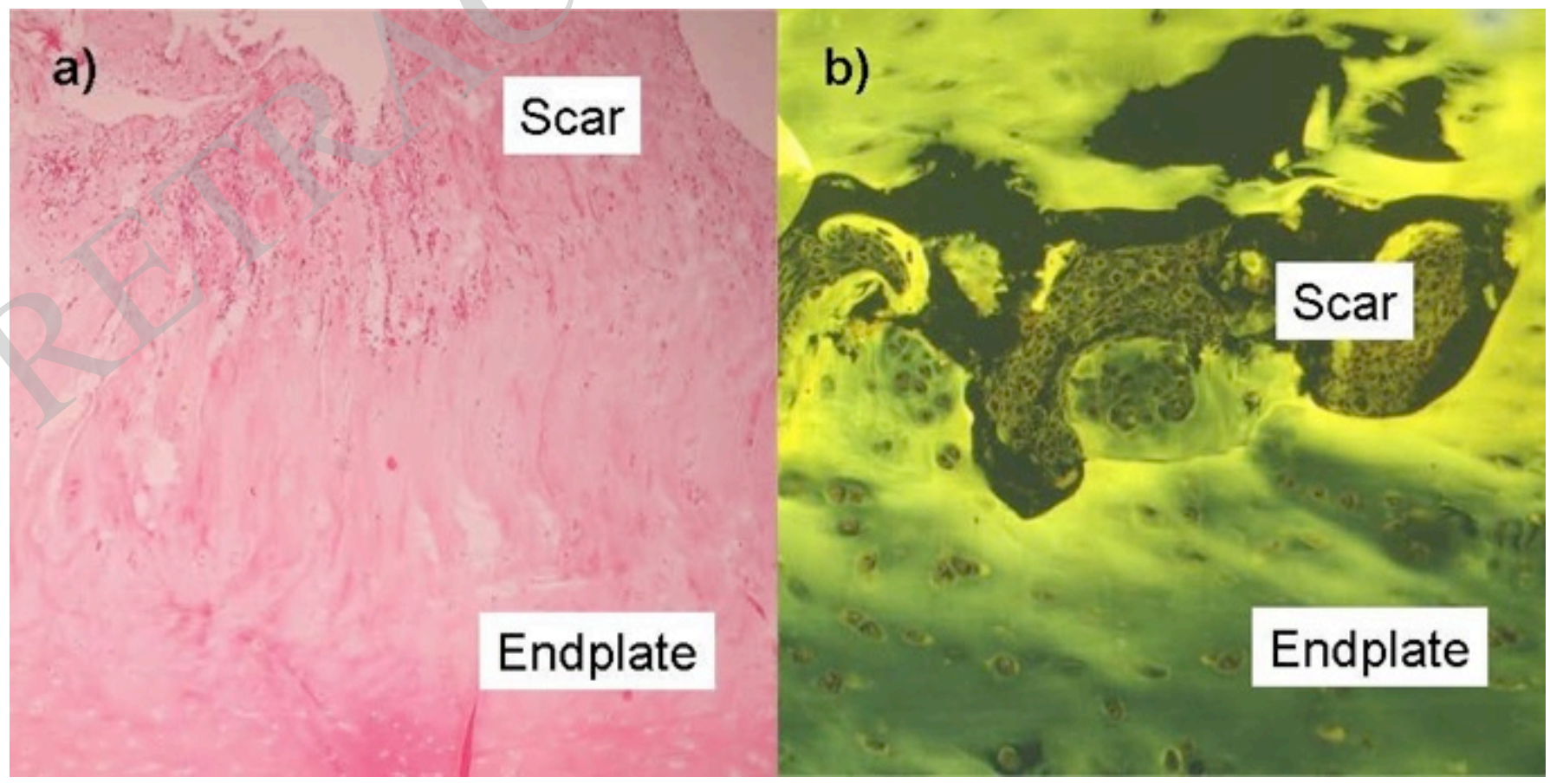

Fig. (5). Part a): The upper part displays granular tissue representing the scar. The lower part shows intact hyaline endplate cartilage. HE, 100x. Part b): Scar formation in an endplate cleft. HE, UV $407 \mu \mathrm{m}, 200 x$. 
a) Chondrocyte regeneration

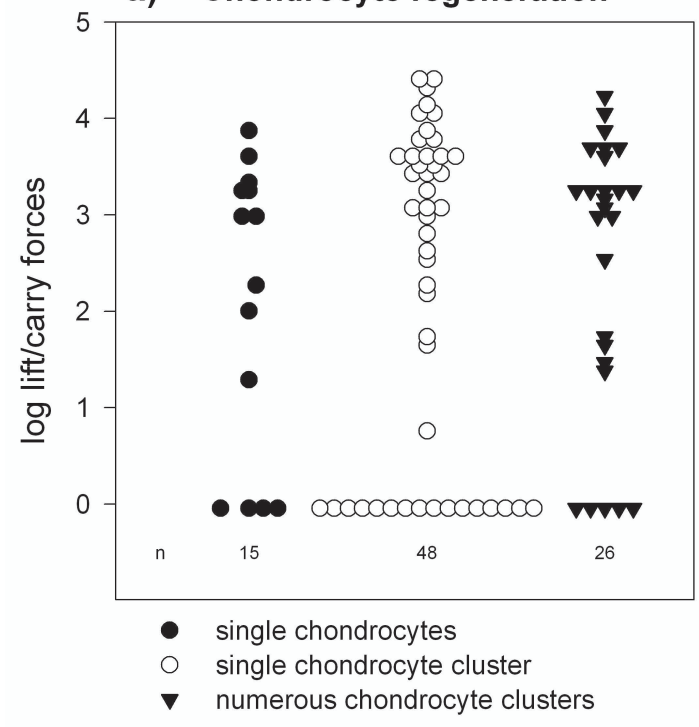

c)

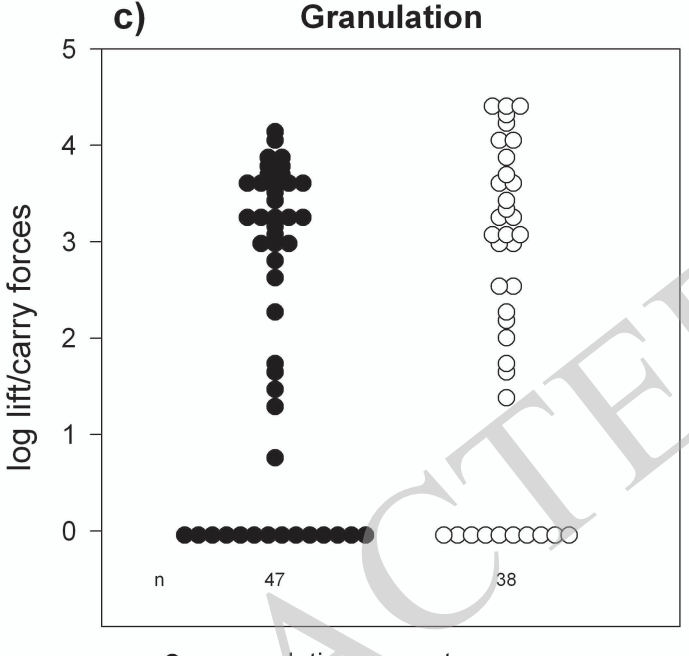

b) Degeneration

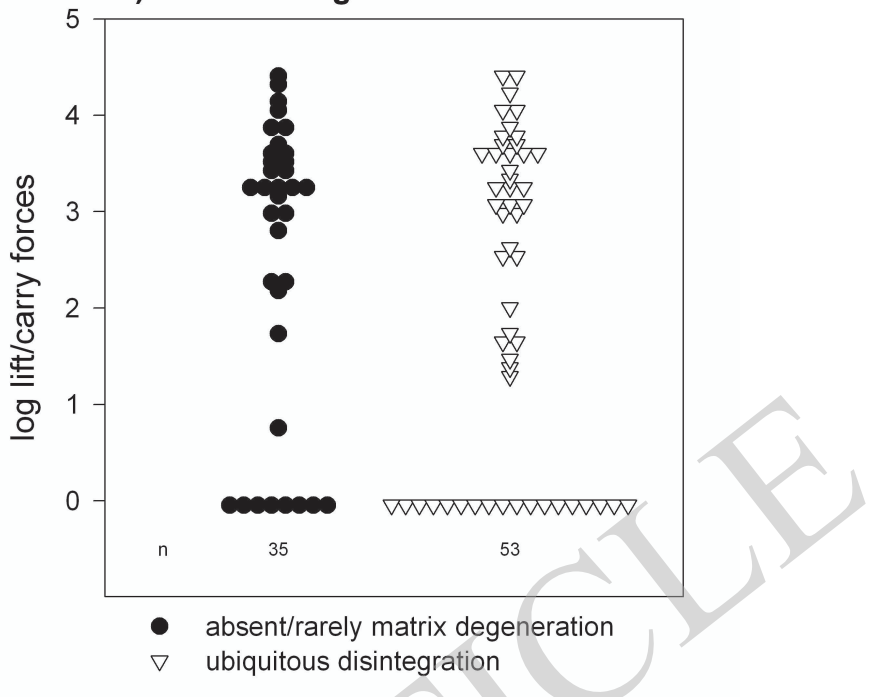

- granulation present

- no granulation

Fig. (6). Cumulative workload data and histological data for patients with lumbar intervertebral disc prolapse. The figure shows lift/carry forces $\left(\log\right.$ of $\left.\mathrm{kg}^{*} \mathrm{~h}\right)$ and histological data. No association was found between total lifetime workload and histology. a) chondrocyte regeneration, b) degeneration and c) scar formation.

histological parameters showed no correlation with histological findings.

In the past disc degeneration was considered to be caused by mechanical stress [14]. Genetically based studies changed the view of determinants of lumbar disc degeneration [13]. The current model of disc degeneration pathogenesis pronounces the effect of heredity and is supported by well conducted twin studies. The effect of heredity is considered to be about $30-50 \%$ [13]. Currently, genetic and environmental influences on disc degeneration seem to be of equal importance. Considering our results showing no association between occupational workload and histological findings our data support the notion of the importance of heredity. Putting the results of the EPILIFT [8] and ours together the combination of occupational workload and genetic factors (not shown in our study) may well contribute to disc prolapse $[9,10]$.

The assessment of cumulative workload was based on the structured interview with about 500 questions. The choice of questions, the assessment of workload of more than $5 \mathrm{~kg}$, the minimum work time of 6 months and other features of the occupational history are based on empirical research [15]. The interpretation of workload data usually follows different models. There are no objective data for validating workload dose models. The EPILIFT study has revealed that different dose models and the primary work load data show a similar qualitative outcome with differences in individual quantitative results [8]. The interviewer bias was reduced by choosing intensively trained lay persons who had been trained in standardized interview techniques [17]. Professional interviewers were chosen for the follow-up data 
Table 3. Crosstab of patients with or without workload exposure during the year before surgery.

\begin{tabular}{|c|c|c|c|}
\hline \multirow{2}{*}{$\begin{array}{c}\text { Workload Exposure } \\
\text { During the Year Before Surgery }\end{array}$} & \multicolumn{3}{|c|}{ Chondrocyte Clusters } \\
\cline { 2 - 4 } & No Clusters (Single Chondrocytes) & Single Chondrocyte Clusters (> 5 Clusters) & Numerous Chondrocyte Clusters \\
\hline \hline yes & $3(10.7 \%)$ & $12(42.9 \%)$ & $13(46.4 \%)$ \\
\hline no & $12(19.7 \%)$ & $36(59.0 \%)$ & $13(21.3 \%)$ \\
\hline
\end{tabular}

The crosstab shows the number and percentage ( $\%$ of the row) of patients with and without workload exposure during the year before surgery. Patients without workload ( $=61)$ had less chondrocyte cluster formation than patients with a high workload $(n=28) ; \mathrm{p}=0.055$ Chi-Square Likelihood quotient.

reported in the EPILIFT study. Data from professional interviewers were not used in this study.

A weakness of epidemiological data collection is the selection bias in favor of disc prolapse patients. Those patients who subjectively suffered most were selected. The selection of patients with objective neurological deficits for this study is seemingly objective. In fact many disc patients with similar objective neurological deficits, e.g. paralysis, did not choose surgical therapy for various reasons. Disc surgery is elective, and a motor deficit does not oblige either the patient or the doctor to choose surgical therapy. There is even a tendency to avoid surgery in the presence of a mild motor deficit [18].

Patients with workload exposure during the last year before surgery grew more chondrocyte clusters than patients without such exposure. This may support the assumption that exposure to heavy workloads is a factor that triggers regenerative processes in lumbar discs. This concept follows the idea of animal experiments on disc trauma proving that different traumata initiate pathological disc changes with subsequent cellular and matrix reactions. The situation in human pathology is not clear, since it is not known which of the tissue changes are due to physiological ageing and which changes come about through non-physiological dise trauma [19]. It is generally assumed in epidemiological studies that workload has a negative influence on the disc. Only the extent of this relationship is under discussion in occupational medicine [20]. Our data does not support the notion of the impact of workload on the microscopic structure in terms of degeneration and scar formation. This raises the question as to whether workload triggers selective effects on disc changes. Our data suggests that the workload may be a potential cofactor like genetic features or lifestyle but that it is not the main cause of significant disc disease or even prolapse.

Relevant chondrocyte proliferation during the last year before surgery was the only histological finding that correlated with workload. The specific investigation of the clusters revealed patterns of recent chondrocytic activity. The clusters started with a few cells and a small neo-matrix rim and were highly positive for the growth factor BMP 2/4. They developed to a certain size related the surrounding matrix and then stopped growing. Large clusters showed a higher rate of pycnic cells and are interpreted as clusters that had grown in the past (Figs. 1, 2). Our BMP expression studies support this view, since the cells in small chondrocyte clusters were vital and metabolically active. These observations are similar to those of investigations using the 3-H-thymidine labeling method that showed chondrocyte clusters to appear as a indicator of proliferation [21].

A period of one year prior to surgery was selected on the basis of the clinical observation that symptoms appeared approximately 6 months to 1 year before surgery in the patient group (own studies). The time correlation between workload and chondrocyte proliferation remained vague within this one year period. Three-dimensional chondrocyte clusters grew in vitro to a size of approximately $1 \mu \mathrm{m}$ in about 1 week. After 3 months, proliferation decreased and matrix production increased [22]. The in vitro data support an estimated time span of less than one year for growth of a chondrocyte cluster.

Chondrocyte cluster formation in arthritic degeneration of big joints shows a similar kind of growth, aging and disintegration [23]. The mechanisms of growth control and cell death remain unclear. We offer the argument that the growth of the surrounding matrix halo and its interconnection with the adult matrix increases the diffusion barrier and subsequently reduces the nutrition of the cluster cells. We found felt-like matrix thickening around some of the clusters, which would support this view. The clusters in this study reached a median area size of $6.50 \mu \mathrm{m}$. Large clusters were associated with a high cell number. Cluster formation in human joints displays comparable size and growth patterns [23]. Disc chondrocyte clusters grow to a similar size in vitro and stop growing due to unknown intrinsic mechanisms [22]. It is accepted that chrondrocyte cluster formation appears in the vicinity or on the surface of ruptured collagen matrix and is considered a general repair phenomenon for large joints [23]. We offer the opinion that this behavior is not just a secondary phenomenon but a quantitatively relevant process. Our study shows that about $30 \%$ of the cases displayed relevant amounts of new chondrocyte tissue, and we showed disintegration of the cluster and the formation of intra-discal sequester which may start the processes of sequestration.

Fibroblast derived scars or granulation tissue are generated after endplate cracks that allow blood cells from the vertebral body to invade the intradiscal space. Fibroblast derived scars are the entrance zone of blood vessels and sensory nerve growth. These pathological changes are suspected to be one reason for discogenic pain [24]. In our specimens, scars were observed in approximately half of the cases. We counted even small scars (present/absent), which may be a reason for the large number compared to the literature. The high frequency of scar formation in the annulus area may increase disc degeneration and may play a role in the generation of disc prolapse. The MR studies of Rasajekaran show that endplate cracks leading to fibroblast 
scars happen more frequently than observed in autopsy studies, even in juvenile persons without known workload or trauma [25]. Our results showed that scar formation did not correlate with workload.

One limitation of our study is the use of MR images in radiologic quantification of degenerative disc changes. There is a number of scoring systems or quantitative MR methods. According to Modic et al. is the value of MRI of the lumbar spine confounded by the high prevalence of morphologic changes in the asymptomatic population [26]. Furthermore our data on spinal histology are rather qualitatively assessed. To our opinion and in accordance with literature and histopathological experience detailed quantification of degeneration of disc tissue would eligible but it is not clear of a specific metric value improves the evaluation of histologic material.

\section{CONCLUSION}

The major finding of the study was that workload had no detrimental effect on the microanatomy of the nuclear and annular matrix of lumbar discs. The only observable histological reaction was an increase in chondrocyte repair activity around annular clefts. It remained unclear whether the clefts appeared due to workload or whether the chondrocyte activation was an independent process triggered in the vicinity of existing clefts. On this basis, workload cannot be identified as a factor determining disc prolapse.

\section{CONFLICT OF INTEREST}

The authors confirm that this article content has no conflict of interest.

\section{ACKNOWLEDGEMENTS}

The study was funded by the German Federation of Institutions for Statutory Accident Insurance and Prevention (HVBG).

\section{REFERENCES}

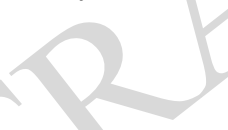

[1] Brandenburg S. Erfahrungen der Berufsgenossenschaften mit der Umsetzung der Berufskrankheit Nr. 2108. 1998; 3-17.

[2] Bolm-Audorff U. Gesicherte Erkenntnisse und Probleme der Epidemiologie der LWS-Erkrankung bei verschiedenen Berufsgruppen. 1998; 34-50.

[3] Hill AB. The environment and disease: Association or causation? Proc R Soc Med 1965; 58: 295-300.

[4] Videman T, Nurminen M, Troup JD. 1990 Volvo Award in clinical sciences. Lumbar spinal pathology in cadaveric material in relation to history of back pain, occupation, and physical loading. Spine 1990; 15: 728-40.

[5] Guiot B, Fessler R. Molecular biology of degenerative disc disease. Neurosurgery 2000; 47: 1034-42.

[6] Mohr W. Morphologie des organisierten Bindegwebes. Gelenkpathologie: Historische Grundlagen, Ursachen und
Entwicklungen von Gelenkleiden und ihre Pathomorphologie 2000; 44-53.

[7] Nerlich A, Boos N, Wiest I, Aebi M. Immunolocalization of major interstitial collagen types in human lumbar intervertebral discs of various ages. Virchows Arch 1998; 432(1): 67-76.

[8] Seidler A, Bergmann A, Jäger M, et al. Cumulative occupational lumbar load and lumbar disc disease--results of a German multicenter case-control study (EPILIFT). BMC Musculoskelet Disord 2009; 10: 48 .

[9] Ala-Kokko L. Genetic risk factors for lumbar disc desease. Ann Med 2002; 34: 42-7.

[10] Virtanen IM, Karppinen J, Taimela S, et al. Occupational and genetic risk factors associated with intervertebral disc disease. Spine 2007; 32: 1129-34.

[11] Nachemson A, Schultz A, Berkson M. Mechanical behavior of human lumbar spine motion segments: influence of age, sex, disc level, and disc degeneration. Spine 1979; 4: 1-8.

[12] Boos N, Rieder R, Schade V, Spratt KF, Semmer N, Aebi M. 1995 Volvo Award in clinical sciences. The diagnostic accuracy of magnetic resonance imaging, work perception, and psychosocial factors in identifying symptomatic disc herniations. Spine 1995; 20: 2613-25.

[13] Battie MC, Videman T, Levalahti E, Gill K, Kaprio J. Genetic and environmental effects on disc degeneration by phenotype and spinal level: a multivariate twin study. Spine (Phila Pa 1976 ) 2008; 33: 2801-8.

[14] Battie MC, Videman T, Kaprio J, et al. The twin spine study: contributions to a changing view of disc degeneration. Spine $\mathrm{J}$ 2009; 9: 47-59.

[15] Karasek R, Brisson C, Kawakami N, Houtman I, Bongers P, Amick B. The Job Content Questionnaire (JCQ): an instrument for internationally comparative assessments of psychosocial job characteristics. J Occup Health Psychol 1998; 3: 322-55.

[16] Richter P, Hemmann E, Merboth H, Fritz S, Hansgen C, Rudof M. Das Erleben von Arbeitsintensität und Tätigkeitsspielraum Entwicklung und Validierung eines Fragebogens zur orientierenden Analyse (FIT). Z Arbeits-Organisation-Psychol 2000; 44: 129-39.

Wynder EL. Investigator bias and interviewer bias: the problem of reporting systematic error in epidemiology. J Clin Epidemiol 1994; 47: 825-7.

[18] Peul WC, van Houwelingen HC, van den Hout WB, et al. Surgery versus prolonged conservative treatment for sciatica. N Engl J Med 2007; 356: 2245-56.

[19] Rabischong P, Louis R, Vignaud J, Massare C. The intervertebral disc. Anat Clin 1978; 1: 55-64.

[20] Battie MC, Videman T, Gibbons LE, Fisher LD, Manninen H, Gill K. 1995 Volvo Award in clinical sciences. Determinants of lumbar disc degeneration. A study relating lifetime exposures and magnetic resonance imaging findings in identical twins. Spine 1995; 20: 2601-12.

[21] Rötzer A, Mohr W. 3H-thymidin-inkorporation in chondrozyten arthrotischen knorpels. Z Rheumatol 1992; 51: 172-6.

[22] Anderer U, Libera J. In vitro engineering of human autogenous cartilage. J Bone Miner Res 2002; 17: 1420-9.

[23] Mohr W. Reaktionsformen des organisierten Bindegewebes 2000; 1: 113-20.

[24] Aoki Y, Akeda K, An H, et al. Nerve fiber ingrowth into scar tissue formed following nucleus pulposus extrusion in the rabbit anularpuncture disc degeneration model: effects of depth of puncture. Spine 2006; 31: E774-80.

[25] Rajasekaran S, Babu J, Arun R, Armstrong B, Shetty A, Murugan S. ISSLS prize winner: A study of diffusion in lumbar discs: a serial magnetic resonance imaging study documenting the influrence of the endplate on diffusion in normal and degenerate discs. Spine 2004; 29: 2654-7.

[26]. Modic MT, Ross JS. Lumbar degenerative disk disease. Radiology 2007; 245: 43-61. 